\title{
Statistical Modeling of Pairs of Sulci in the Context of Neuroimaging Probabilistic Atlas
}

\author{
Isabelle Corouge and Christian Barillot \\ Projet Vista, IRISA/INRIA-CNRS, Rennes, France \\ \{icorouge, cbarillo\}@irisa.fr \\ http://www.irisa.fr/vista
}

\begin{abstract}
In the context of neuroimaging probabilistic atlases, we propose a statistical framework to model the inter-individual variability of pairs of sulci with respect to their relative position and orientation. The approach extends previous work [3], and relies on the statistical analysis of a training set. We first define an appropriate data representation, through an observation vector, in order to build a consistent training population, on which we then apply a normed principal components analysis (normed-PCA). Experiments have been performed on pairs of major sulci extracted from $18 \mathrm{MR}$ images.
\end{abstract}

Keywords: Neuroimaging, probabilistic atlases, cortical sulci, statistical modeling, normed-PCA.

\section{Introduction}

This paper comes within the context of digital cerebral probabilistic atlases. We are particularly interested in the study of inter-individual variability of cortical structures (sulci/gyri) which are of major interest both from an anatomical as well as a functional point of view. This paper pursues previous work [3] where we proposed a statistical modeling of cortical sulci shapes and of their variations, as well as a consistent way to use it for functional data inter-individual registration purpose. We aim now at modeling relationships between major sulci in terms of their position and orientation. Thus, the final model will present a hierarchical aspect by discrimination of different types of variations, with on the one hand shape variations, and on the other hand, position and orientation variations.

To grasp the high inter-individual variability implied by the studied data, we use a deformable model [10] of "active shape models" type [2], [4]. Thus, we learn the variability of the considered class of objects on a training population, and can then deduce occurrence probabilities of the studied structures. Some authors have also used point distribution models (PDMs) to model sets of cortical sulci (e.g. [1] and [9]). Our approach differs first by its matching scheme which is a very simple one. It consists in positioning oneself in a local scope in which instances to learn are naturally matched. Then, we rely on a parametric representation of the sulci which describes not only their external traces but also their buried part. This leads to a more complete model of the inter-individual cortical variability, all the more so as the buried part represents, at least, two thirds of the cortex. In this sense, our approach relates to [7], but still differs since 


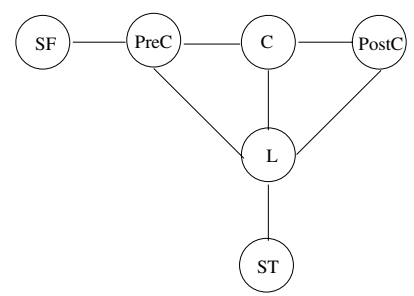

Fig. 1. Relation graph between major sulci for one hemisphere. Six major sulci are considered: superior frontal sulcus (SF), precentral sulcus (PreC), central sulcus (C), postcentral sulcus (PostC), lateral sulcus or sylvian fissure (L) and superior temporal sulcus (ST).

the authors perform a prior global registration towards stereotactic system and do not analyze sulci interactions. At last, our approach is based on a graph structure describing the involved sulci as nodes, and their relationships as arcs (see Fig. 1). This aspect can be related to works [6] and [8] where authors build a graph devoted to automatic (or semi-automatic) labeling of cortical sulci.

As work is still in progress, we present here the statistical modeling of a reduced graph, and consider presently a pair of sulci and its variations in terms of position and orientation. After a brief remind of some necessary preprocessing stages in Sect. 2, we present the statistical modeling of a pair of sulci in Sect. 3. First, we define the observations (or individuals) constituent of the training population, i.e. we define an appropriate data representation to build the training set. Second, we analyze these observations by the mean of a statistical analysis, the normed principal components analysis (normed-PCA). In Sect.4, we present experiments and discuss them as well as the approach in Sect. 5] before concluding in Sect. 6

\section{Preprocessing}

The representation of sulci used in this paper, as well as the representation of the position and orientation within a brain of one sulcus, result from previous work, [5], [3], which we briefly recall here. Sulci are defined as their median surface and extracted from MRI volumes by a method now known as the "active ribbon" method [5]. It leads to a parametric representation of the sulci by cubic B-spline surfaces. The spline, parameterized by $u$ and $v$, is described by $n b c=n b c_{-} u * n b c_{-} v$ control points where $n b c_{-} u$ (resp. $n b c_{-} v$ ) is the number of control points in the direction associated with parameter $u$ (resp. $v$ ). The parametric direction $u$ represents the length of the sulcus and the direction $v$ its depth. The position and orientation within a brain of one sulcus is then represented by a coordinate system, local to this sulcus [3]. The origin of this local coordinate system, specifying the position of the sulcus, is defined as the center of mass of the sulcal surface. The three axes, specifying the orientation of the sulcus are defined as its axes of inertia. The first axis follows the length of the sulcus and is oriented from foot to head. The second one follows the depth of the sulcus and is oriented toward the outside of the brain. The third axis, orthogonal to the two first ones, is then the normal to the regression plane of the sulcus, and follows the antero-posterior direction in case of left sulci (see Fig. 2), and postero-anterior direction in case of right sulci. 


\section{Statistical Modeling of Pairs of Sulci}

\subsection{Training and Data Representation}

Learning the variability of a class of objects over a set of observations requires first of all to define what is an observation vector for this class of objects. Since the orientation and position of one sulcus within the brain is represented by its local coordinate system, we encode the relative position and orientation of two sulci by the pair of coordinate systems local to these sulci. However, the local system is dependent of the coordinate system in which the sulcus is initially expressed; and this coordinate system differs from one subject to another one. To not be limited by this dependence and, by this way, be able to match the observations as requires by the statistical analysis, we define a coordinate system, local to a pair of sulci that we call "local median coordinate system", with respect to which we define the observation vector. Thus, by positioning oneself in a local scope, the observations are consistent over the training population to achieve the statistical analysis.

Local Median Coordinate System. We consider the pair of sulci $(A, B)$.

Let $\mathcal{R}_{A}\left(O_{a}, \mathbf{x}_{a}, \mathbf{y}_{a}, \mathbf{z}_{a}\right)$, resp. $\mathcal{R}_{B}\left(O_{b}, \mathbf{x}_{b}, \mathbf{y}_{b}, \mathbf{z}_{b}\right)$, be the local coordinate system to the sulcus $A$, resp. $B$. Let $\mathcal{R}_{M}\left(O_{m}, \mathbf{x}_{m}, \mathbf{y}_{m}, \mathbf{z}_{m}\right)$ be the local median system. First, the origin $O_{m}$ is defined as the middle of $\left[O_{a} O_{b}\right]$. Next, axis $\mathbf{z}_{\mathbf{m}}$ is defined as:

$$
\mathbf{z}_{m}=\frac{\mathbf{z}_{a}+\mathbf{z}_{b}}{\left\|\mathbf{z}_{a}+\mathbf{z}_{b}\right\|}
$$

and is then the normal of a plane $\pi$. Then $\mathbf{x}_{m}$ and $\mathbf{y}_{m}$ are deduced from the projections, $\mathbf{x}_{p}$ and $\mathbf{y}_{p}$, of $\left(\mathbf{x}_{a}+\mathbf{x}_{b}\right)$ and $\left(\mathbf{y}_{a}+\mathbf{y}_{b}\right)$ on $\pi$. Let $\alpha$ be the angle between $\mathbf{x}_{p}$ and $\mathbf{y}_{p}$ in the plane $\pi$ oriented by $\mathbf{z}_{m}$, and let $\beta=(\pi-\alpha) / 2$. Then:

$$
\left\{\begin{array}{l}
\mathbf{x}_{m}=\mathbf{R}_{/ \mathbf{z}_{\mathbf{m}},-\beta}\left(\mathbf{x}_{p}\right) \\
\mathbf{y}_{m}=\mathbf{R}_{/ \mathbf{z}_{\mathbf{m}}, \beta}\left(\mathbf{y}_{p}\right)
\end{array}\right.
$$

where $\mathbf{R}_{/ \mathbf{z}_{m}, \beta}$ is the matrix of the rotation defined by the axis $\mathbf{z}_{m}$ and the angle $\beta$. Such a rotation matrix is easily obtained thanks to the Rodrigues formula. Thus, for a given axis, $\mathbf{n}$, and a given angle, $\omega$ :

$$
\mathbf{R}_{/ \mathbf{n}, \omega}=\mathbf{I}+\sin (\omega) \boldsymbol{\Gamma}(\mathbf{n})+(1-\cos (\omega)) \boldsymbol{\Gamma}^{2}(\mathbf{n})
$$

where $\mathbf{I}$ is the identity matrix and $\boldsymbol{\Gamma}(\mathbf{n})$ is the vector product matrix, i.e.:

$$
\boldsymbol{\Gamma}(\mathbf{n})=\left(\begin{array}{ccc}
0 & -n_{z} & n_{y} \\
n_{z} & 0 & -n_{x} \\
-n_{y} & n_{x} & 0
\end{array}\right)
$$

Definition of the Observation Vector. Rather than directly use the expression of $\mathcal{R}_{A}$ and $\mathcal{R}_{B}$ in $\mathcal{R}_{M}$ as the observation vector, we have preferred to describe the relative orientation and position of $(A, B)$ by the parameters of the transformation bringing $\left(\mathcal{R}_{A}, \mathcal{R}_{B}\right)$ towards $\mathcal{R}_{M}$. It is in fact, more suitable to perform a statistical analysis later 
on. We define then such a transformation, enabling to locate an orthonormal basis with respect to another one.

Let $\mathcal{B}_{f}\left(\mathbf{x}_{f}, \mathbf{y}_{f}, \mathbf{z}_{f}\right)$ and $\mathcal{B}(\mathbf{x}, \mathbf{y}, \mathbf{z})$ be two orthonormal bases. Then, considering the basis $\mathcal{B}_{f}$ as fixed, to apply a composition of three rotations defined by the axis of $\mathcal{B}_{f}$ and appropriate angles, $\psi, \phi$ and $\theta$, transforms $\mathcal{B}$ towards $\mathcal{B}_{f}$ :

$$
\mathcal{B}_{f}=\mathbf{R}_{\mathbf{z}_{f}, \theta} \mathbf{R}_{/ \mathbf{y}_{f}, \phi} \mathbf{R}_{/ \mathbf{x}_{f}, \psi}(\mathcal{B})
$$

The angles $\psi, \phi$ and $\theta$, which are in fact Euler angles, are defined as following through their cosines and sines:

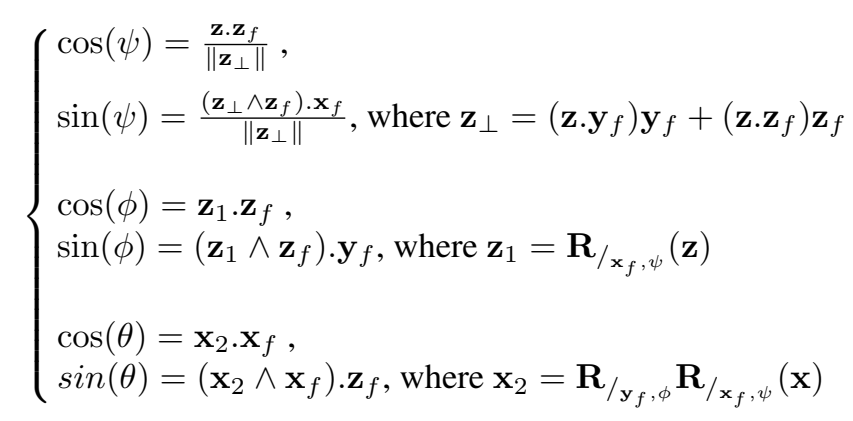

where "." denotes scalar product and " $\wedge$ " vector product.

Thus computing the six Euler angles $\psi_{\mathcal{R}_{A}}, \phi_{\mathcal{R}_{A}}, \theta_{\mathcal{R}_{A}}$ and $\psi_{\mathcal{R}_{B}}, \phi_{\mathcal{R}_{B}}, \theta_{\mathcal{R}_{B}}$ defined such that:

$$
\left\{\begin{array}{l}
\left(\mathbf{x}_{m}, \mathbf{y}_{m}, \mathbf{z}_{m}\right)=\mathbf{R}_{/ \mathbf{z}_{m}, \theta_{\mathcal{R}_{A}}} \mathbf{R}_{/ \mathbf{y}_{m}, \phi_{\mathcal{R}_{A}}} \mathbf{R}_{/ \mathbf{x}_{m}, \psi_{\mathcal{R}_{A}}}\left(\mathbf{x}_{a}, \mathbf{y}_{a}, \mathbf{z}_{a}\right) \\
\left(\mathbf{x}_{m}, \mathbf{y}_{m}, \mathbf{z}_{m}\right)=\mathbf{R} / \mathbf{x}_{m}, \theta_{\mathcal{R}_{B}} \mathbf{R}_{/ \mathbf{y}_{m}, \phi_{\mathcal{R}_{B}}} \mathbf{R}_{/ \mathbf{x}_{m}, \psi_{\mathcal{R}_{B}}}\left(\mathbf{x}_{b}, \mathbf{y}_{b}, \mathbf{z}_{b}\right)
\end{array}\right.
$$

enables to completely define the orientation of sulci $A$ and $B$ with respect to $\mathcal{R}_{M}$. As the position is concerned, we also use Euler angles to determine it. Let $\psi_{O_{a}}, \phi_{O_{a}}$ and $\theta_{O_{a}}$ be the Euler angles defining the direction of $\overrightarrow{O_{m} O_{a}}$. They are defined such that:

$$
\mathbf{z}_{m}=\mathbf{R}_{/ \mathbf{z}_{m}, \theta_{O_{a}}} \mathbf{R}_{/ \mathbf{y},_{m},{ }_{O_{a}}}{\mathbf{R} / \mathbf{x}_{m}, \mathcal{O}_{a}}\left(\frac{\overrightarrow{O_{m} O_{a}}}{\left\|\overrightarrow{O_{m} O_{a}}\right\|}\right)
$$

Note that, in this case, $\theta_{O_{a}}=0$. Accordingly only $\psi_{O_{a}}$ and $\phi_{O_{a}}$ are used to characterize the direction of vector $\overrightarrow{O_{m} O_{a}}$. Let $d$ be the distance between $O_{m}$ and the origins of the local systems, $d=\left\|\overrightarrow{O_{m} O_{a}}\right\|=\left\|\overrightarrow{O_{m} O_{b}}\right\|$. Then knowing $d, \psi_{O_{a}}, \phi_{O_{a}}$ enables to completely define the position of sulcus $A$ with respect to $\mathcal{R}_{M}$. The position of sulcus $B$ with respect to $\mathcal{R}_{M}$ is similarly computed and characterized by $d, \psi_{O_{b}}$ and $\phi_{O_{b}}$.

Eventually, the observation vector, encoding the relative orientation and position between two neighboring sulci is:

$$
\mathbf{e}=\left(d, \psi_{O_{a}}, \phi_{O_{a}}, \psi_{O_{b}}, \phi_{O_{b}}, \psi_{\mathcal{R}_{A}}, \phi_{\mathcal{R}_{A}}, \theta_{\mathcal{R}_{A}}, \psi_{\mathcal{R}_{B}}, \phi_{\mathcal{R}_{B}}, \theta_{\mathcal{R}_{B}}\right)
$$

Note that for a given e, the origin and the axis of $\mathcal{R}_{A}$ and $\mathcal{R}_{B}$ can be completely recovered since (2) is reversible:

$$
\mathcal{B}=\mathbf{R}_{/ \mathbf{x}_{f},-\psi} \mathbf{R}_{/_{\mathbf{y}_{f},-\phi}} \mathbf{R}_{/ \mathbf{z}_{f},-\theta}\left(\mathcal{B}_{f}\right)
$$




\subsection{The Normed-PCA}

To analyze the variations in orientation and position between two sulci over a population, and by this mean, model the inter-individual variability at the level of the considered couple, we use a principal component analysis (PCA). Such an analysis reveals in fact the main modes of variation relatively to a mean observation. It enables to represent data in a new basis, also orthogonal, but which supresses the redundancy of information of the original data in the sense that, variables in the new basis are not correlated. Since the vector e characterizing the relative position and orientation of two sulci is not homogeneous (i.e. its first element is a distance, whereas the other ones are angles), we use a normed-PCA. It is an appropriate analysis technique when variables do not have same unit. As a matter of fact, the normed-PCA consists in diagonalizing the centered and normed data covariance matrix, i.e. the correlation data matrix, rather than the original data covariance matrix like PCA does. Thus, the distance between two individuals does not depend on the variables units, and balance between variables is restored since they have then all unit variance.

Analysis. Let $\mathcal{P}$ be the training population made up of $n$ observations $\mathbf{e}_{i}$, defined by $p$ variables. In our case, an observation $\mathbf{e}_{i}$ is defined by (5) and $p=11$. Let $\mathbf{E}=\left(e_{i j}\right)$ be the $n \times p$ matrix of the observations, and $\overline{\mathbf{e}}$ be the mean observation vector, $\overline{\mathbf{e}}=\frac{1}{n} \sum_{i=1}^{i=n} \mathbf{e}_{i}$. Let $\mathbf{D}_{1 / \sigma}$ be the $p \times p$ diagonal matrix of the inverse standard deviations $\sigma_{j}$ of the original variables, $\sigma_{j}=\frac{1}{n} \sum_{i=1}^{i=n}\left(e_{i j}-\bar{e}_{j}\right)^{2}$. Then, the matrix $\mathbf{X}$ of centered-normed data is:

$$
\mathbf{X}=(\mathbf{E}-\mathbf{1} \overline{\mathbf{e}}) \mathbf{D}_{1 / \sigma}
$$

where 1 is a $n \times 1$ vector having all its elements equal to 1 . Diagonalizing the covariance matrix, $\mathbf{C}$, of the centered-normed data provides the new basis $\mathbf{U}$ :

$$
\mathbf{C}=\frac{1}{n} \mathbf{X}^{t} \mathbf{X}=\mathbf{U} \boldsymbol{\Lambda} \mathbf{U}^{t}, \text { where } \boldsymbol{\Lambda}=\operatorname{diag}\left(\lambda_{1}, \ldots, \lambda_{p}\right) \text { with } \lambda_{1} \geq \lambda_{2} \geq \ldots \geq \lambda_{p}
$$

In this new basis, the observations are expressed as: $\mathbf{F}=\mathbf{X U}$, what leads to the following reconstruction formula:

$$
\mathbf{e}_{i}=\overline{\mathbf{e}}+\mathbf{f}_{i} \mathbf{U}^{t} \mathbf{D}_{1 / \sigma}^{-1}, \quad i=1, \ldots, n
$$

Synthesis. Relation (7) can be used to generate new instances of the studied class of objects.

First, since the eigenvalue $\lambda_{j}$ represents the variance along the the $j^{\text {th }}$ mode, a modal approximation can be achieved by retaining only the first $t,(t \leq p)$, eigenvectors of the modal basis $\mathbf{U}$. The quality of the approximation can be measured by the proportion, $\tau$, of the whole variance, $\lambda_{T}$, explained by the retained modes:

$$
\tau=\frac{\sum_{j=1}^{j=t} \lambda_{j}}{\lambda_{T}} \text { where } \lambda_{T}=\sum_{j=1}^{j=p} \lambda_{j}
$$


Second, under the assumption that the distribution of centered-normed observations $\mathbf{x}_{i}$ is gaussian, i.e $\mathbf{x}_{i} \sim \mathcal{N}(0, \mathbf{C})$, it comes that $\mathbf{f}_{i} \sim \mathcal{N}(0, \boldsymbol{\Lambda})$ and $\mathbf{f}_{i j} \sim \mathcal{N}\left(0, \lambda_{j}\right)$. Accordingly, new instances consistent with the learnt observations can be synthesized, by the variation of $\mathbf{f}_{i j, j=1, \ldots, t}$ in a suitable range, which is typically such that:

$$
-3 \sqrt{\lambda_{j}} \leq \mathbf{f}_{i j} \leq+3 \sqrt{\lambda_{j}}
$$

In fact, if $\mathbf{f}_{i j} \sim \mathcal{N}\left(0, \lambda_{j}\right)$, then $P\left(\mathbf{f}_{i j} \leq 3 \sqrt{\lambda_{j}}\right)=99.7 \%$, and thus (8) can be considered as a condition of representativity of the class of objects of interest.

\section{Experiments}

Our database is made up of 18 subjects $(35+/-10$ years old healthy males, all righthanded) who have underwent a T1-MR SPGR 3D study. Six major sulci have been extracted for each hemisphere. They are superior frontal sulcus (SF), precentral sulcus (PreC), central sulcus (C), postcentral sulcus (PostC), lateral sulcus or sylvian fissure (L) and superior temporal sulcus (ST). For each of them, local coordinate systems have been computed (see Fig. 2). Statistical modeling experiments have been performed on pairs $(\mathrm{C}$, PreC), (C, PostC) and (L, ST) of the left hemisphere.

We can on see on Fig.2 the 3 local median coordinate systems corresponding to these pairs of sulci. In Table 1, we exhibit the percentage of cumulative variance explained along the modes of variations. The variations due to the first, as well as those due to the third mode mode are illustrated on Fig. 3 for the three considered pairs of sulci.
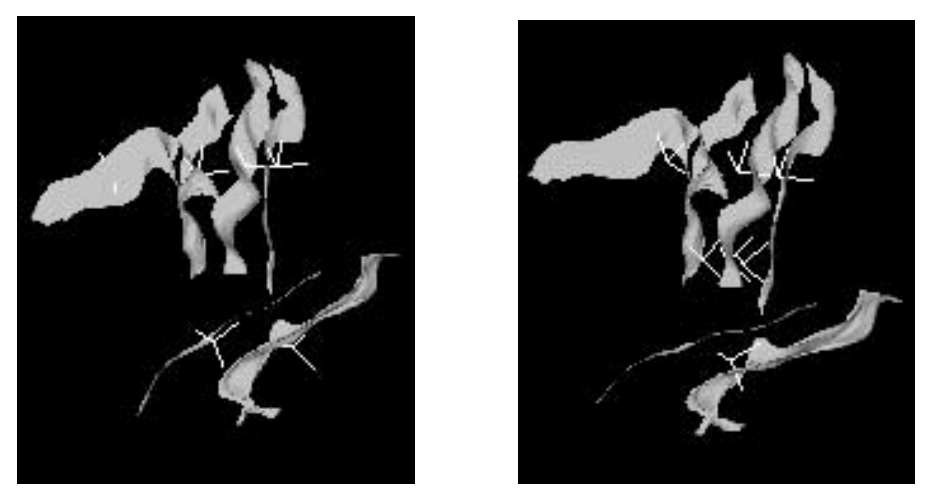

Fig. 2. For both figures, the 6 sulci are extracted from one subject and are, from left to right and then top to bottom: SF, PreC, C, PostC, L, ST, of the left hemisphere. Left: a view of the 6 considered major sulci with their local systems of coordinate superimposed. Right: a view of the 6 considered major sulci with the local median system of each pair superimposed.

\section{Discussion}

First, we remark on Table 1 that for each experimented pair, the whole variance is explained by 7 modes whereas original data are expressed by 11 variables. As a matter 
Table 1. Percentage, $p$, of cumulative variance according to the number of modes retained, $t$, for pairs (C, PreC), (C, PostC) and (L, ST); $p=\frac{\sum_{j=1}^{j=t} \lambda_{j}}{\lambda_{T}} \times 100$.

\begin{tabular}{|c||c|c|c|}
\hline & $(\mathrm{C}$, PreC $)$ & $(\mathrm{C}$, PostC $)$ & $(\mathrm{L}, \mathrm{ST})$ \\
\hline \hline 1 & 48.844 & 42.5621 & 36.7084 \\
\hline 2 & 79.105 & 68.697 & 61.9032 \\
\hline 3 & 90.5307 & 87.5777 & 84.3016 \\
\hline 4 & 97.1499 & 95.1251 & 94.5972 \\
\hline 5 & 99.0582 & 98.3103 & 98.3764 \\
\hline 6 & 99.9343 & 99.6695 & 99.9023 \\
\hline 7 & 100 & 100 & 100 \\
\hline
\end{tabular}
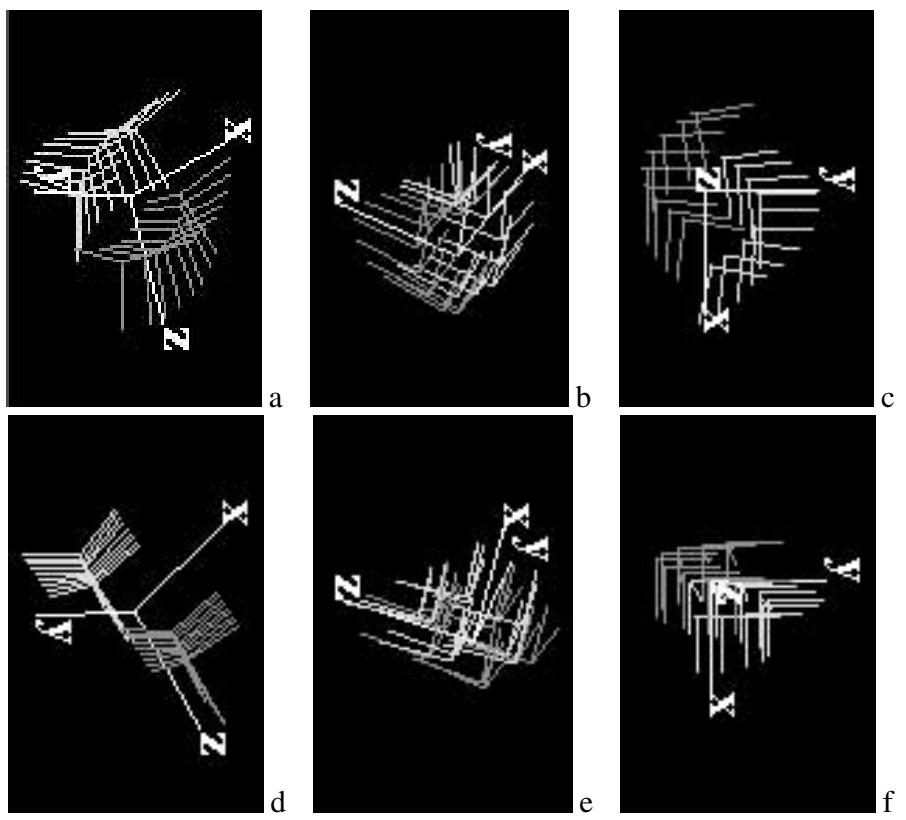

Fig. 3. Top row: variations of the first mode around the mean observation: $-2 \sqrt{\lambda_{1}} \leq \mathbf{f}_{1} \leq$ $+2 \sqrt{\lambda_{1}}$. Bottom row: variations of the third mode around the mean observation: $-2 \sqrt{\lambda_{3}} \leq \mathbf{f}_{3} \leq$ $+2 \sqrt{\lambda_{3}}$. For all figures: white: local median system, dark gray: synthesized local coordinates systems of sulcus $A$ around its mean coordinate system, light gray: synthesized local coordinates systems of sulcus $B$ around its mean coordinate system.

a, d: pair (preC, C); b, e: pair (C, postC); c, f: pair (L, ST).

of fact, the observation vector e contains some redundant information since 4 angles out of the 10 ones used in this vector can be expressed, by construction, as a linear combination of the other ones (i.e. $\psi_{\mathcal{R}_{B}}=-\psi_{\mathcal{R}_{A}}, \phi_{\mathcal{R}_{B}}=-\phi_{\mathcal{R}_{A}}, \psi_{O_{b}}=\psi_{O_{a}}-\pi$ and $\left.\phi_{O_{b}}=-\phi_{O_{a}}\right)$. Second, modeling the variability of the pairs of sulci we considered in the experiments is relevant by itself since these quasi-parallel sulci define gyri. For 
example, the central and postcentral sulci bound the postcentral gyrus, and modeling their interaction is a way of modeling the gyrus variability. As the other arcs are concerned, since they represent some "plis de passage", their modeling will rather find its interest in the study of the whole graph.

\section{Conclusion}

We have proposed a statistical framework to model the inter-individual variability of pairs of sulci with respect to their relative position and orientation. The modeling is performed by a modal analysis (normed-PCA) on a consistent training population. Work in progress aims first at extending the inter-individual fusion scheme proposed in [3]. This one consists in registering functional activitions under the constraint of anatomical landmarks: the sulci. Until now, the constraint was limited to one sulcus, whereas the functional are located in one gyrus, and in this sense are under the influence of the two sulci bounding this gyrus. So we will use the statistical modeling presented here to introduce a constraint extended to a pair of sulci. Second, we intend to extend the proposed modeling framework to the whole graph.

\section{References}

1. Caunce, A., Taylor, C.J.: Building 3D Sulcal Models Using Local Geometry. Medical Image Analysis, 5:69-80 (2001)

2. Cootes, T.F., Taylor, C.J., Cooper, D.H., Graham, J.: Active Shape Models - their training and application. CVIU, 61(1):38-59 (1995)

3. Corouge, I., Barillot, C., Hellier, P., Toulouse, P., Gibaud, B.: Non-linear local registration of functional data. MICCAI, LNCS, 2208:948-956 (2001)

4. Kervrann, C., Heitz, F.: A hierarchical statistical framework for the segmentation of deformable objects in image sequences. IEEE CVPR, 724-728 (1994)

5. Le Goualher, G., Barillot, C.,Bizais, Y.: Modeling cortical sulci with active ribbons. IJPRAI, 8(11):1295-1315 (1997)

6. Le Goualher, G., Procyk, E., Collins, L., Venegopal, R., Barillot C., and Evans, A.: Automated extraction and variability analysis of sulcal neuroanatomy. IEEE Trans. on Medical Imaging, 18(3):206-217 (1999)

7. Le Goualher, G., Argenti, A.-M., Duyme, M., Baare, W.F.C., Hulshoff Pol, H.E., Barillot, C., Evans, A.C.: Statistical sulcal shape comparisons: application to the detection of genetic encoding of the central sulcus shape. NeuroImage, 11(5):564-574 (2000)

8. Rivière, D., Mangin, J-F., Papadopoulos, D., Martinez, J-M., Frouin, V., Régis, J.: Automatic Recognition of cortical culci using a congregation of neural networks. MICCAI, LNCS, 1935:40-49 (2000)

9. Tao, X., Han, X., Rettmann, M.E., Prince, J.L., Davatzikos, C.: Statistical study on cortical sulci of human brains. IPMI, LNCS, 2082:475-487 (2001)

10. McInerney, T., Terzopoulos, D.: Deformable Models in Medical Image Analysis: A Survey. Medical Image Analysis, 1(2):91-108 (1996) 